\title{
Structure Retrieval of Strongly Scattering Materials in the Transmission Electron Microscope
}

\author{
H. G. Brown ${ }^{1 *}$, Z. Chen ${ }^{2}$, M. Weyland ${ }^{3,4}$, C. Ophus ${ }^{1}$, J. Ciston ${ }^{1}$, L. J. Allen ${ }^{5}$ and S. D. Findlay ${ }^{6}$ \\ 1. National Centre for Electron Microscopy (NCEM), Molecular Foundry, Lawrence Berkeley National \\ Laboratory, Berkeley, USA \\ 2. School of Applied and Engineering Physics, Cornell University, Ithaca, USA \\ 3. Monash Centre for Electron Microscopy (MCEM), Monash University, Melbourne, Australia \\ 4.Department of Materials Science and Engineering, Monash University, Melbourne, Australia \\ 5. School of Physics, the University of Melbourne, Melbourne, Australia \\ 6. School of Physics and Astronomy, Monash University, Melbourne, Australia \\ * Corresponding author: hamishbrown@lbl.gov
}

With the ability to form sub-Ångstrom sized beams and the electron beam's sensitivity to very small amounts of material, the electron microscope is a powerful tool for materials scientists and biologists. With recent developments in detectors, direct and efficient visualization of specimen features such as long range electric and magnetic fields and light atomic columns are now routine in STEM using differential phase contrast (DPC) and ptychographic reconstruction.

To achieve this, most reconstruction methods such as DPC and ptychography simplify the complex and subtle physics of electron scattering, which generally requires a (scattering) matrix solution, Fig. 1(a), by assuming a specimen much thinner than the electron probe depth of field, this is known as the "projection approximation", Fig. 1(b). With an appropriate choice of probe forming aperture such an approximation is typically valid for low resolution work, such as imaging nanometer scale electric and magnetic fields, even for samples 100s of nanometers thick [1]. For atomic resolution analysis, where a finely converged (thus small depth of field) probe is required, the projection approximation has a limited range of validity, restricting quantitative reconstruction to samples thinner than about $30 \AA[2,3]$.

We demonstrate how to overcome this limitation by reconstructing the scattering matrix from experimental data. We first record a focal series in STEM, Fig. 2(a), using an FEI CETA fast-readout electron camera to capture the full diffraction pattern for each probe position (ie. a focal series of 4D STEM scans). We use a $230 \AA$ silicon sample oriented down the [110] axis for this proof of principle experiment. Using small virtual diffraction plane detectors, Fig. 2(b), we synthesize a focal series of bright field STEM images, Fig. 2(c), and perform iterative focal series wave function reconstruction on each of these series. The resulting set of complex wave functions, shown in Fig. 2(d), is a representation of the scattering matrix. Using some known, general symmetries of this matrix we can solve for the Fourier coefficients of the specimen electrostatic potential [4]. The resulting reconstruction is improved relative to that achieved in DPC, as shown in Fig. 2(e), however experimental artifacts, including spatial incoherence of our STEM instrument and residual aberrations of our probe forming lens limit the quality of our reconstructions [5]. With the next generation of microscopes coming online these limitations can be more easily overcome and lead to routine reconstruction of thick and strongly scattering samples [6].

\section{References:}


[1] H. G. Brown et al., Ultramicroscopy 182, (2017) p 169.

[2] R. Close et al., Ultramicroscopy 159, (2015) p 124.

[3] K. Müller-Caspary et al., Ultramicroscopy 178, (2017) p 62.

[4] L. J. Allen et al., Acta Crystallographica Section A 56, (2000) p 119.

[5] H. G. Brown et al., Physical Review Letters 121, (2018) 266102.

[6]This research was supported under the Australian Research Council Discovery Projects funding scheme (Project No. DP140102538). HGB and JC acknowledge additional funding form the DOE Early Career Research Program. Work at the Molecular Foundry was supported by the Office of Science, Office of Basic Energy Sciences, of the U.S. Department of Energy under Contract No. DE-AC0205CH11231. Thermo Fisher Scientific are acknowledged for experimental high speed 4D-STEM acquisition software.

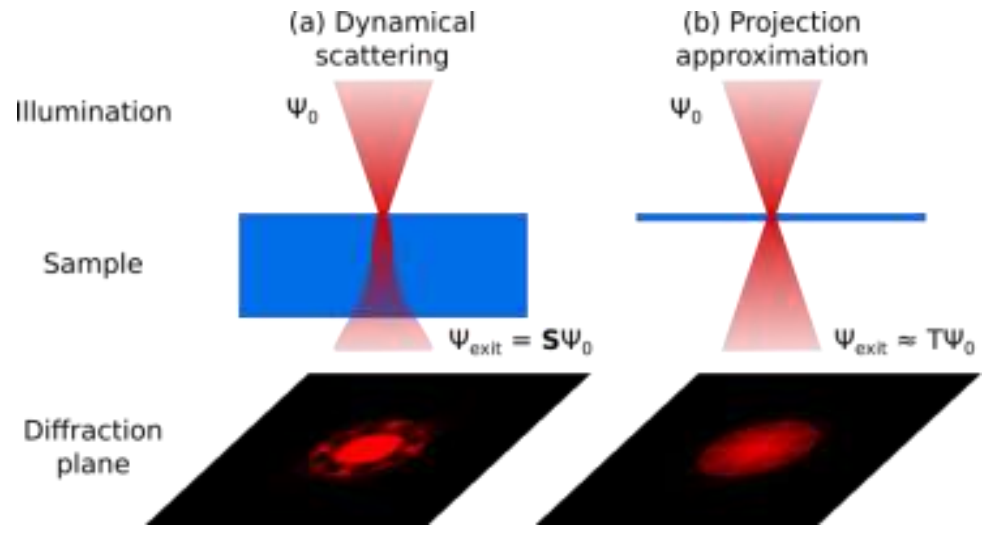

Figure 1. (a) In general the scattering of a fast electron (incident wave $\psi_{0}$ ) must be modeled using a scattering matrix $(\mathbf{S})$. (b) The projection approximation assumes a very thin specimen, resulting in a

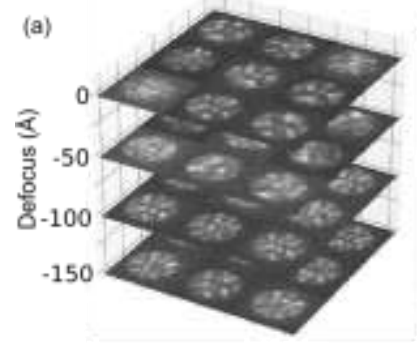

(d)

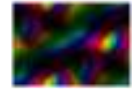

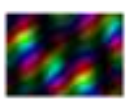
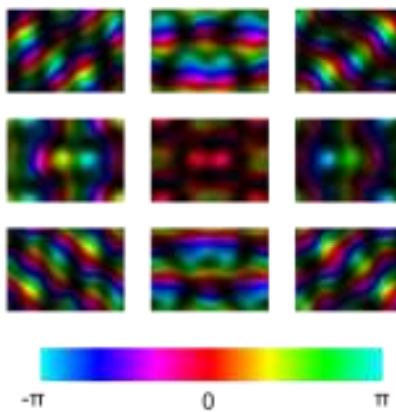

(c)
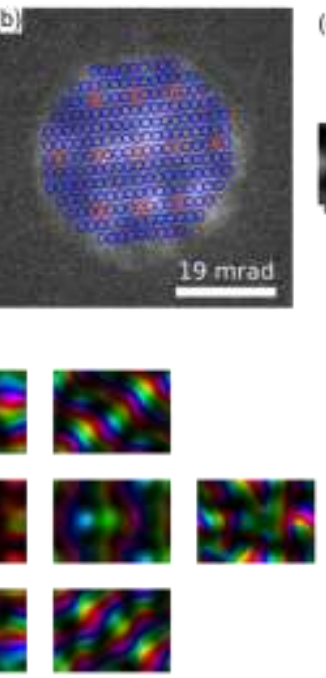

(e)
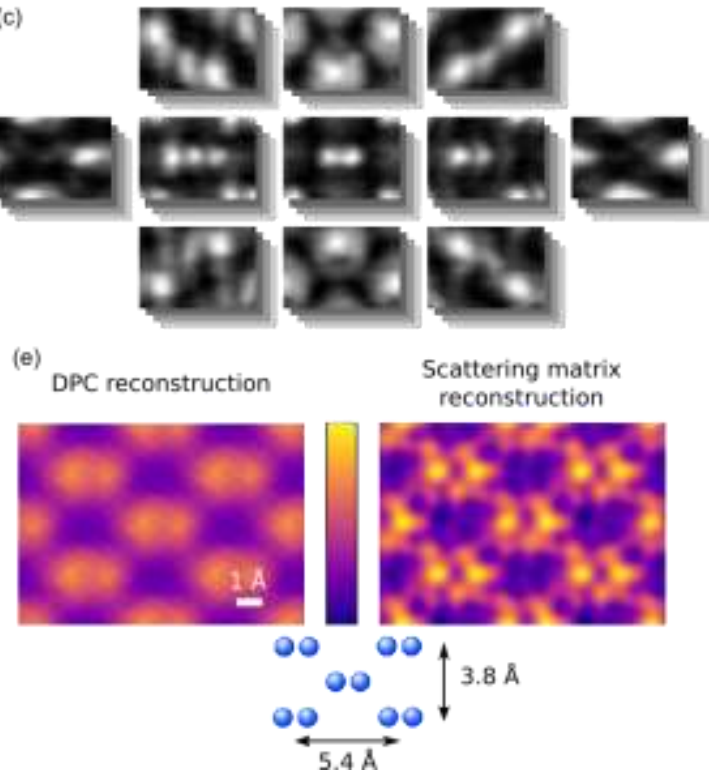

simpler model where the illumination is multiplied by a specimen transmission function $\mathrm{T}$ in real space . Figure 2. A scattering matrix reconstruction from a focal series of STEM data show in (a). Images are synthesized from the diffraction patterns in (b) and a montage of these images is shown in (c). Iterative wave function reconstruction is performed on each image set - results are shown in (d) - and the crystalline structure, shown in (e), is solved using the method in ref. [4] with a DPC reconstruction shown for comparison. 